\title{
O silêncio no cotidiano do adolescente com HIV/AIDS
}

\author{
Silence in the daily life of the adolescent with HIVIAIDS \\ El silencio en el cotidiano del adolescente con VIH/SIDA
}

\section{Maria da Graça Corso da Motta', Eva Neri Rubim Pedro', Cristiane Cardoso de Paula", Débora Fernandes Coelho"II, Aline Cammarano Ribeiro" ${ }^{\mathrm{IV}}$, Aramita Prates Greff",

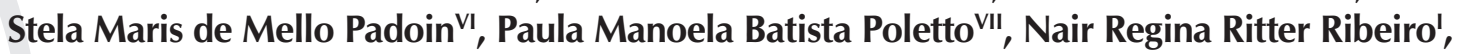 Helena Becker Issi ${ }^{i v}$, Eliane Tatsch Neves ${ }^{\mathrm{VI}}$, Neiva Isabel Raffo Wachholz ${ }^{\mathrm{VIII}}$, Regis Kreitchmann ${ }^{\mathrm{IX}}$, Aline Goulart Krue ${ }^{\mathrm{VII}}$}

'Universidade Federal do Rio Grande do Sul, Escola de Enfermagem, Departamento de Enfermagem Materno-Infantil, Programa de Pós-Graduação em Enfermagem. Porto Alegre-RS, Brasil. "Universidade Federal de Santa Maria, Centro de Ciências da Saúde, Departamento de Enfermagem. Santa Maria-RS, Brasil. "I' Universidade Federal de Ciências da Saúde de Porto Alegre, Departamento de Enfermagem, Curso de Bacharelado em Enfermagem. Porto Alegre-RS, Brasil.

IV Universidade Federal do Rio Grande do Sul, Escola de Enfermagem, Programa de Pós Graduação em Enfermagem (Doutoranda). Porto Alegre-RS, Brasil.

$\checkmark$ Secretária Municipal da Saúde de Porto Alegre. Porto Alegre-RS, Brasil.

V' Universidade Federal de Santa Maria, Centro de Ciências da Saúde, Departamento de Enfermagem, Curso de Graduação em Enfermagem, Programa de Pós Graduação em Enfermagem. Santa Maria-RS, Brasil.

VII Universidade Federal do Rio Grande do Sul, Escola de Enfermagem,

Programa de Pós Graduação em Enfermagem (Mestranda). Porto Alegre-RS, Brasil.

VIII Prefeitura Municipal de Porto Alegre, Secretaria Municipal de Saúde. Porto Alegre-RS, Brasil.

Ix Prefeitura Municipal de Porto Alegre, Secretaria da Saúde. Santa Casa de Misericórdia,

Centro de Pesquisa Materno Infantil da Irmandade. Porto Alegre-RS, Brasil.

Submissão: 19-07-2012 Aprovação: 22-05-2013

\section{RESUMO}

Este estudo caracteriza-se por ser uma pesquisa qualitativa que objetivou desvelar a percepção e a vivência em relação ao tratamento antirretroviral do adolescente com síndrome da imunodeficiência adquirida. A pesquisa foi realizada em serviços de referência em dois municípios na região sul do Brasil. A produção dos dados foi desenvolvida com a dinâmica de criatividade e sensibilidade mapa falante, por cinco participantes. Foi aplicada a técnica de análise temática do conteúdo. Das produções artísticas e depoimentos emergiram o cotidiano do uso dos medicamentos e o silêncio do diagnóstico da doença e do tratamento que implicam o cuidado à saúde. Conclui-se que é necessário o envolvimento dos profissionais de saúde, possibilitando espaços para a família e promovendo diálogos desta com o adolescente, visando à adesão ao tratamento.

Descritores: Saúde do Adolescente; HIV; Síndrome de Imunodeficiência Adquirida; Adesão à Medicação; Antirretroviral de Alta Atividade.

\section{ABSTRACT}

This study is characterized as a qualitative research with the objective of unveiling the perception and experience regarding the antiretroviral treatment of the adolescent with acquired immunodeficiency syndrome. The research was carried out within reference health care centers in two municipalities from the southern region of Brazil. The data production was developed through the creativity and sensitiveness dynamics of talking map type by a group of five participants. One applied the technique of content thematic analysis. The artistic productions and testimonies evidenced their daily experience with use of medications and the silence on the diagnosis of the disease and its treatment that implies the health care. The conclusion drawn is that the involvement of health professionals is needed since it opens spaces for the family besides promoting the dialogue of its members with the adolescent aiming at the adhesion to the treatment.

Key words: Adolescent's Health; HIV; Acquired Immunodeficiency Syndrome; Adhesion to Medication. High Activity Antiretroviral. 


\section{RESUMEN}

Se trata de una pesquisa cualitativa que intenta revelar la percepción y experiencia vivida con relación al tratamiento antirretroviral del adolescente con síndrome de inmunodeficiencia adquirida. Se realizó la pesquisa en servicios de salud de referencia, en dos municipalidades de la región sur de Brasil. La producción de los datos fue desarrollada a través de la dinámica de creatividad y sensibilidad de mapa hablante, por un grupo de cinco participantes. Se lo aplicó la técnica de análisis temático de contenido. De las producciones artísticas y testimonios, emergieron el cotidiano del uso de medicamentos y el silencio acerca del diagnóstico de la enfermedad y de su tratamiento que implican el cuidado a la salud. Se concluye que es necesario el envolvimiento de los profesionales de salud porque abre espacios para la familia y promueve diálogos de sus miembros con el adolescente visando la adhesión al tratamiento.

Palabras clave: Salud del Adolescente; VIH; Síndrome de Inmunodeficiencia Adquirida; Adhesión a la Medicación; Antiretroviral de Alta Actividad. 


\section{INTRODUÇÃO}

Com a evolução da AIDS no Brasil evidencia-se uma tendência de juvenização da epidemia, marcada pelos casos notificados por idade, ou seja, aumento da distribuição dos casos entre adolescentes. No período de 1980-2011 ocorreram 12.891 casos na faixa etária entre 13 a 19 anos $^{(1)}$. Essa mudança implicou a formação de políticas específicas e o avanço do tratamento, resultando na melhoria e bem-estar dessa população.

Os avanços do tratamento desecadeiam o cotidiano terapêutico permeado por vivências que decorrem do uso permanente de medicamentos, da frequência às consultas, da realização de exames de rotina e demais cuidados para a manutenção de sua saúde ${ }^{(2)}$, e, ainda somam-se as dificuldades quanto à revelação do diagnóstico para o adolescente ${ }^{(3-5)}$.

Quando o diagnóstico não é revelado predomina o silêncio nas famílias e surgem os desafios relativos aos cuidados com a saúde que influenciam a adesão. Portanto, realizar o tratamento pode ser uma forma de não anunciar a existência da doença, pois não haverá manifestação dos sintomas, e o adolescente não sofrerá consequências, por exemplo, o preconceito. Já, o silêncio limita o acesso aos direitos sociais e de saúde ${ }^{(6-7)}$.

Percebe-se, portanto, que as vivências do adolescente centram-se nas dimensões clínica e social, as quais repercutem na adesão ao tratamento antirretroviral (TARV). O uso sistemático dos medicamentos específicos objetiva a melhora clínica, o aumento do tempo de vida e a redução de falhas terapêuti$\operatorname{cas}^{(8-9)}, \mathrm{e}$, para tanto, é importante uma aliança terapêutica entre o profissional e adolescente, podendo-se incluir a família que lhe proporciona no cotidiano.

A partir dessa problemática buscou-se desenvolver um estudo Multicêntrico nos municípios de Porto Alegre e Santa Maria, estado do Rio Grande do Sul, intitulado Impacto de Adesão ao tratamento antirretroviral em crianças e adolescentes, na perspectiva da família, da criança e do adolescente, sob o contrato $n^{\circ}$ : ED03756/2006(UNESCO); TRPJ $n^{\circ}$ As -3833/2006, financiado pelo Departamento de HIV/AIDS e Hepatites Virais do Ministério da Saúde, Organização das Nações Unidas para a Educação, Ciência e a Cultura.

A pesquisa foi desenvolvida nesses dois municípios por serem centros de referência para o atendimento das Doenças Sexualmente Transmissíveis (DSTs) e HIV/AIDS das proximidades regionais. Desenvolvida em duas fases - quantitativa e qualitativa - nessa pesquisa pretendeu-se desvelar a percepção e a vivência do adolescente que tem AIDS, em relação ao tratamento antirretroviral.

\section{MÉTODO}

Pesquisa de cunho qualitativo, com etapa de campo desenvolvida em serviços especializados em DST/AIDS no município de Porto Alegre e de Santa Maria, no estado do Rio Grande do Sul. Em Porto Alegre, contemplou o Serviço de Assistência Especializada em DSTs e AIDS, o Serviço de Atenção Terapêutica do Hospital Sanatório Partenon e o Grupo de Atenção à AIDS Pediátrica do Ambulatório de Pediatria, Hospital da Criança Conceição. Em Santa Maria, contemplou o Serviço de Doenças Infecciosas Pediátricas do Hospital Universitário de Santa Maria.

Os sujeitos do estudo atenderam aos critérios de inclusão: faixa etária de 13 a 19 anos de idade, com diagnóstico de AIDS, segundo critérios do Departamento DST/AIDS e Hepatites Virais do Ministério da Saúde; e em tratamento com antirretroviral com, pelo menos, três meses.

Na produção dos dados foram utilizadas as Dinâmicas de Criatividade e Sensibilidade (DCS), fundamentadas no Método Criativo e Sensível (MCS). Esse Método é subsidiado pela pedagogia crítico-reflexiva de Freire. A partir da Tese de Doutoramento da Professora e Enfermeira Ivone Evangelista Cabral, de 1997, passou-se a utilizar as DCS, uma alternativa para a pesquisa em enfermagem, considerando-se que um objeto de estudo valoriza as singularidades de cada participante do grupo e pela coletivização das experiências. A DCS propõe um espaço de discussão coletiva, em que a experiência vivenciada é abordada por meio de uma produção artística ${ }^{(10)}$.

O Método Criativo e Sensível ocorreu em cinco momentos: no primeiro fez-se a apresentação de cada membro do grupo concomitante com a integração e a interação dos participantes e a pesquisadora. No segundo momento foram disponibilizados materiais para a realização da dinâmica, com as questões norteadoras: as facilidades e dificuldades em relação ao Tratamento Antirretroviral (TARV) e o que foi feito para superá-las. Foi desenvolvida a DCS Livre para Criar com grupo de adolescentes com o objetivo de desvelar a vivência dos adolescentes em relação ao uso de antirretrovirais em seu cotidiano. Neste estudo, essa DCS teve como questão geradora do debate com o grupo de adolescentes: Como é para ti tomar o remédio sempre, todos os dias? No terceiro, os participantes apresentaram as suas produções artísticas individuais ou coletivas, socializando-se o que foi produzido. Os temas geradores codificados foram negociados com os participantes, que, no quarto momento, os decodificaram em subtemas durante a análise coletiva e a discussão grupal. Por fim, no quinto momento realizou-se a síntese temática dos temas e subtemas e a validação dos dados ${ }^{(10)}$.

Em relação à logística da pesquisa, os encontros foram previamente planejados, considerando-se a escolha de um local apropriado e a previsão dos materiais a serem disponibilizados. Os materiais utilizados foram crachás de identificação dos participantes, cadeiras dispostas ao redor de uma mesa, gravador de fita cassete, folhas de cartolina, canetas e lápis coloridos e folhas de papel A4.

Nas três oficinas realizadas, com duração aproximada de cinquenta minutos a uma hora e trinta minutos, as DCS foram coordenadas por um dos pesquisadores responsáveis pelo projeto e contaram com alunos da graduação e pós-graduação na função de auxiliares de pesquisa.

Para a análise dos dados foi utilizada a técnica de Análise Temática do Conteúdo que consiste em descobrir núcleos de sentido, cuja presença ou frequência seja expressiva para o objetivo analítico visado. Na pré-análise foram organizadas as informações com uma leitura. A partir da exploração do material, buscaram-se os significados e os agrupamentos das informações, surgindo os temas ou categorias ${ }^{(11)}$. Para tanto, 
foi desenvolvido um quadro analítico, do qual emergiram as categorias: o cotidiano medicamentoso e o silêncio do adolescente que tem HIV/AIDS. Na última etapa fez-se a análise e a interpretação das facetas das percepções dos adolescentes, discutidas com a produção científica da temática.

Os cinco adolescentes, com idade entre 11 e 14 anos, foram selecionados a partir da primeira etapa dessa pesquisa multicêntrica, a qual foi aprovada pelos Comitês de Ética em Pesquisa (CEP) das instituições envolvidas: protocolos de aprovação 2005446 (CEP/UFRGS), 23081.017341/2006-61 (CEP/UFSM), 001014268.07.8 (CEP/SMS/PORTO ALEGRE ) e 113/08 (CEP/GHC).

As questões éticas cumpriram com a proteção dos participantes quanto aos princípios de: voluntariedade, anonimato, confidencialidade das informações da pesquisa, justiça, equidade, diminuição dos riscos e potencialização dos benefícios, resguardando sua integridade física-mental-social de danos temporários e permanentes. O termo de consentimento livre esclarecido foi assinado pelo responsável legal e o próprio adolescente assinou o termo de assentimento.

\section{RESULTADOS E DISCUSSÃO}

O relato dos adolescentes demonstrou a percepção sobre a AIDS relacionada a horário, a ingestão do medicamento fora de casa, a quantidade, ao tamanho, ao gosto do remédio, ao uso prolongado e aos efeitos adversos.

[...] Esse remédio líquido também enjoa o estômago [...] sempre [...] Depois que a gente tomar o remédio tem que comer alguma coisa senão a gente fica mal [...]o remédio é muito forte [...] sente enjoo se tu não come. A gente tem que tomar e comer na hora (A1).

É ruim por causa do horário que tem que ter horário pra tomar, ou às vezes quando não tem aula eu durmo até tarde, mas eu tenho que me acordar e tomar e depois tentar dormir de novo [...] é difícil [...] (o remédio) faz bem para nossa saúde, que ajuda na nossa saúde [...] (A2).

[...] A minha mãe fez até isso (lembrou) para eu não tomar remédio na hora da escola [...] Vai se acostumando a tomar remédio [...] Eu tomo 4 ou 5 remédios [...]Não é bom ter que tomar muito remédio, ter que tomar bastante é ruim [...] (A3).

A terapia medicamentosa possui características quanto à quantidade de $A R V$, apresentação, horários e frequência, o que implica a não adesão ${ }^{(12)}$. Os medicamentos de uso contínuo são definidos como uma "prisão" para as pessoas com HIV/aids cujas vidas estão diretamente ligadas aos horários, esquemas e dosagens exigidos pelo TARV. Além disso, a complexidade da terapêutica medicamentosa interfere na adaptação do cotidiano dessas pessoas quanto às reformulações na alimentação, horários e ritmo diário para cumprir o tratamento ${ }^{(13)}$.

Para tomar os ARV o adolescente e sua família buscam estratégias para terem doses fora do horário da escola. $\mathrm{O}$ adolescente toma o ARV nos períodos em que está em casa, o que possibilita a privacidade em seu tratamento e a manutenção do sigilo do diagnóstico. Tomar o medicamento fora do domicílio revela-se uma dificuldade relacionada a fatores sociais e estilo de vida, pois, muitas pessoas com a infecção necessitam esconder a medicação de amigos e familiares ${ }^{(14)}$. Algumas pessoas utilizam estratégias para lidar com as dificuldades: escondem o medicamento, retiram rótulos de frascos, tomam os ARV em lugares seguros (para que as pessoas que não tenham conhecimento da doença não os vejam na hora de tomá-los). Essas atitudes apontam para o medo da discriminação(13).

Fatores associados às dificuldades de tomada de medicamentos incluem: a intolerância ao cheiro e ao gosto, tamanho, quantidade e uso prolongado. A apresentação dos medicamentos e suas características referentes à cor, ao odor, ao gosto, tamanho, às embalagens são determinantes para a não adesão dos $A R V^{(8,14)}$. Apesar do avanço do TARV, esses fatores fragilizam a continuidade do tratamento, pois, muitos evitam tomar os medicamentos no intuito de preservar seu bem-estar.

Estudos sobre o tema revelam, além desses fatores, outras condições associadas à baixa adesão de pessoas que fazem uso de ARV: complexidade do esquema, forma de armazenamento, número de doses, horários das doses, que podem gerar conflito com as rotinas e o estilo de vida(15). Isso resulta em dificuldades para as pessoas se adequarem às necessidades do TARV ao cotidiano, por exemplo, horários de acordar, das refeições, da escola e/ou do trabalho ${ }^{(16-18)}$.

$\mathrm{O}$ uso prolongado de $\mathrm{ARV}$, como a situação vivida pelos adolescentes infectados na infância, está relacionado ao acompanhamento clínico permanente e no longo prazo, que pode facilitar o processo de adaptação e aceitação dos esquemas de ARV. Entretanto, o tempo indeterminado do tratamento dessa doença crônica, ainda sem cura, possivelmente implica a dificuldade de adesão(19).

Mesmo com os avanços da terapia de medicamentos para o HIV/aids, os efeitos colaterais dos ARV são frequentes e podem se associar aos fatores relacionados à fragilidade na adesão ao TARV, pois os principais efeitos são: diarreia, distúrbios gastrintestinais (vômito e náuseas) e rash cutâneo ${ }^{(2,13)}$.

Ressalta-se a importância do entendimento e da compreensão dos adolescentes sobre a função do medicamento em sua vida, da terapia proposta e seus possíveis efeitos adversos. Isso implica, de maneira direta, na aceitação ao tratamento e na boa aderência. A sensibilização do adolescente sobre a importância do TARV conduz à minimização do impacto dos efeitos adversos, os quais geram reclamações, pois, o adolescente diz que quando não tomava os ARV não sentia desconfortos físicos ${ }^{(16,18)}$. No entanto, o uso do medicamento profilático associado aos ARV é essencial à manutenção da saúde e bem-estar, devido ao comprometimento imunológico, e, também, possibilita minimizar os efeitos adversos no intuito de fortalecer a adesão ao TARV ${ }^{(2,16)}$.

Em suas falas, os adolescentes, participantes do estudo em tela, relataram a presença desse silêncio em suas vidas, destacando-se, aqui, os silêncios no que tange à escola e aos seus pares, caracterizados pelos amigos e colegas de escola.

Nos depoimentos, os adolescentes revelam as pessoas que compõem a rede de relações na escola que sabem de seu 
diagnóstico. É possível supor que a revelação do diagnóstico na escola esteja relacionada à necessidade de as pessoas-chave saberem, a fim de que, caso ocorra algum incidente com o adolescente nas dependências da escola, alguém tome providências. Essas situações apareceram em dois depoimentos, no entanto, considera-se pertinente citá-las, pois os adolescentes, de alguma forma, relatam o silêncio que a doença reproduz e o restrito número de pessoas que conhecem o seu diagnóstico.

Os guris começaram a me chamar de um monte de coisa, mas daí eu nem dou bola [...] eles não sabem só o professor e a diretora (A1).

Meus colegas não sabem; só a professora de educação física e a diretora (A3).

Nesse sentido, os adolescentes com HIV levam sua vida social dentro de uma normalidade peculiar à sua fase do desenvolvimento. Todavia, participar de espaços sociais com outros adolescentes, independente da sorologia, não os coloca à vontade para compartilharem seus diagnósticos com todos, pois há o medo da exclusão do espaço ocupado ${ }^{(20)}$. $\mathrm{Na}$ vida do adolescente, a escola configura-se como uma instituição social muito presente e importante na formação das relações sociais, portanto, silenciar o diagnóstico nesse lugar é bastante comum.

As pessoas com HIV tentam evitar situações que podem gerar preconceito e selecionam com cuidado a quem devem revelar o diagnóstico de sua soropositividade. Assim, evitam atitudes de estigmatização. Os adolescentes identificam algumas situações difíceis na escola, como não se relacionar com os colegas, não participar de certas atividades e não falar sobre seu diagnóstico aos colegas e aos amigos ${ }^{(13,20-21)}$.

É imprescindível refletir sobre as repercussões desse silenciamento na vida dos adolescentes somadas a sua condição de saúde, porque, nessa etapa da vida, a escola é, para a maioria deles, o espaço social mais frequentado. Fica evidente a necessidade de ações que promovam o melhor viver na vida da escola para esses adolescentes que lidam com o medo da discriminação e do rótulo no seu viver.

A revelação entre os pares aparece nos depoimentos dos adolescentes acerca de quem sabe e quem não sabe da sua condição sorológica. Percebe-se que se preocupam e temem a descoberta do diagnóstico por terceiros, devido ao preconceito, situações que podem afetar de modo importante o uso de remédios em locais públicos ou mesmo no domicílio quando estão na presença de outras pessoas, pois os medicamentos podem ser a prova da aids ${ }^{(16,22)}$.

Só um colega meu que sabe, mas [...] é que ele foi lá em casa e escutou [...] ele contou pra mãe e ela não disse nada, que se ele quisesse ficar do meu lado podia (A1).

Um dia um amigo meu foi posar lá em casa, isso faz tempo. Aí ele me viu tomando o remédio e pensou que fosse de laranja e queria tomar também, aí disse que não e ele ficou insistindo e eu tive que esconder o remédio dele (A2).
Compreende-se que esse silêncio é mantido na família e reproduzido pelo adolescente entre seus pares com o objetivo, velado ou não, de não diferenciação e aceitação grupal, como uma característica própria da fase de adolescência. Essa situação vivida está permeada pelo estigma da doença, do preconceito e de atitudes de discriminação que marcam a história da epidemia da Aids ${ }^{(7,20)}$. Também se observa que os adolescentes se preocupam em justificar os motivos pelos quais não deva ocorrer o preconceito. O fato de a infecção ser por categoria de exposição da transmissão vertical, os impulsiona a pensar que a infecção não ocorreu devido a um ato ou situação de vida que eles tiveram algum envolvimento, mas que eles nasceram com isto, portanto, livres de serem julgados.

A mãe me contou que um dia ela foi no pronto-socorro e aí ela viu uma guria de14-16 anos que tinha HIV e o guardinha fez assim: por que tu não se cuidou guria? E a guria disse: por que eu nasci assim, e o guarda começou a rir. Eu não gostei disso, se ele nascesse ele não ia gostar que a gente risse dele (A1).

[...] se é uma pessoa adulta pode muito bem entender, uma criança assim de cinco anos não pode entender, mas uma pessoa adulta assim já é burrice. Porque se a gente nasce assim com isso, a gente não pode evitar; a gente já nasceu (A2).

Não pode humilhar as pessoas que têm. Depois a pessoa fica com vergonha e não quer mais sair (A3).

A história inicial da epidemia contribuiu para esse imaginário coletivo que colocou os grupos de risco como suscetíveis à infecção e, consequentemente, responsáveis por seus atos. No entanto, a história nos mostrou que não existiram grupos de risco e que a epidemia não tem raça, cor, escolaridade, opção e/ou estilo de vida ${ }^{(23)}$. Os protagonistas da epidemia são todos seres humanos que mantêm suas relações sociais no mundo.

O sentido social do preconceito é pejorativo, no qual a opinião do outro acerca de alguém é admitida sem ser discutida, construída a partir de uma visão de mundo acrítica, sendo transmitida culturalmente e, consequentemente, internalizada pelas pessoas, as quais são influenciadas em seus modos de agir ${ }^{(24)}$.

\section{CONSIDERAÇÕES FINAIS}

O adolescente, em seu cotidiano medicamentoso, revela a importância do conhecer acerca de seu tratamento, visando a compreensão sobre a função do medicamento em sua saúde, o que inclui saber os possíveis efeitos adversos e implicações em sua vida. Mostra que mantém o silêncio nos espaços socais que frequenta, em que só algumas pessoas sabem de sua doença, já vivenciou o preconceito e fala da repercussão deste na vida das pessoas que têm a doença. O silêncio se perpetua na família, sendo conservado pelo adolescente com seus pares, com o objetivo, velado ou não, de não diferenciação e aceitação grupal, como uma característica própria da fase de adolescência. Assim, o silêncio na vida do adolescente pode 
conduzir a dificuldades na realização do seu tratamento, ao limitar suas redes de apoio e suas possibilidades de tornar-se protagonista do cuidado de sua saúde.

Destaca-se o envolvimento dos profissionais de saúde que cuidam do adolescente, possibilitando espaços para a família e promovendo diálogos desta com o adolescente, a fim de fortalecer seu bem-estar com sua saúde. Essas ações poderão repercutir na adesão ao tratamento, em situações de preconceitos, e entre outras questões que envolvem a doença, somadas às peculiaridades da adolescência.

\section{REFERÊNCIAS}

1. Ministério da Saúde [homepage na internet]. Programa Nacional de DST e AIDS. Boletim Epidemiológico AIDS/ DST. Versão Preliminar. 2011 [acesso em 02 jun 2012]. Disponível em: http://www.aids.gov.br/sites/default/files/ anexos/publicacao/2011/50652/boletim aids 2011 preliminar3_pdf_20265.pdf

2. Ribeiro AC, Paula CC, Neves ET, Padoin SMM. Perfil clínico de adolescentes que têm aids. Cogitare Enferm [periódico na internet]. 2010 [acesso em 10 jun 2011];15(2):256-62]. Disponível em: http://ojs.c3sl.ufpr. br/ojs2/index.php/cogitare/article/view/17858/11651

3. Lima AAA, Pedro ENR. Crescendo com HIV/aids: estudo com adolescentes portadoras de HIV/AIDS e suas cuidadoras-familiares. Rev Latino-Am Enferm 2008;16(3):348-354.

4. Paula CC, Cabral IE, Souza IEO. O cotidiano de crianças infectadas pelo HIV no adolescer: compromissos e possibilidades do cuidado de si. DST J Bras Doenças Sex Transm 2008;24(3-4):174-179.

5. Paiva SS, Galvão MTG. Gravidez em adolescente com infecção pelo HIV/AIDS. Rev Enferm UERJ 2006;14(4):586-91.

6. Machiesqui SR, Padoin SMM, Paula CC, Ribeiro AC, Langendorf TF. Pessoas acima de 50 anos com aids: implicações para o dia-a-dia. Esc Anna Nery Rev Enferm 2010;14(4):726-731.

7. Gomes AMT, Cabral IE. Ocultamento e silenciamento familiares no cuidado à criança em terapia antiretroviral. Rev Bras Enferm 2010;63(5):719-726.

8. Reiners AAO, Azevedo RCS, Vieira MA, Arruda ALG. Produção bibliográfica sobre adesão/não-adesão de pessoas ao tratamento de saúde. Ciênc Saúde Coletiva [periódico na internet]. 2008 [acesso em 10 jun 2011];13(Suppl.2): 2299-2306. Disponível em: http://www.scielo.br/pdf/csc/ v13s2/v13s2a34.pdf

9. Barroso LLMMB, Pereira KKCP, Almeida PPCA, Galvão MMTGG. Adesão ao tratamento com anti-retrovirais entre pacientes com aids. Online Braz J Nurs [periódico na internet]. 2006 [acesso em 11 jun 2011];5(2). Disponível em: http://www.objnursing.uff.br/index.php/nursing/ article/view/290/56

10. Cabral IE. Método criativo e sensível: alternativa de pesquisa na enfermagem. In: Gauthier JHM, Cabral IE, Santos I, Tavares CMM. Pesquisa em Enfermagem: novas metodologias aplicadas. Rio de Janeiro: Guanabara Koogan, 1998. p. 177-208.

11. Minayo MCS. O Desafio do conhecimento: pesquisa qualitativa em saúde. São Paulo: Hucitec, 2010.

12. Colombrini MRC, Lopes MHBM, Figueiredo RM. Adesão à terapia antiretroviral para o HIV/AIDS. Rev Esc Enferm [periódico na internet]. 2006 Nov [acesso em 11 jun 2011];40(4): 576-81. Disponível em: http://www.ee.usp. $\mathrm{br} /$ reeusp/upload/pdf/292.pdf

13. Texeira MG, Silva GA. A Representação do Portador do Vírus da Imunodeficiência humana sobre o Tratamento com os Anti-retrovirais. Rev Esc Enferm USP 2008;42(4):726-36.

14. Melchior R, Nemes MIB, Alencar TMD, Buchalla CM. Desafios da adesão ao tratamento de pessoas vivendo com HIV/aids no Brasil. Rev Saúde Pública 2007;41(Suppl 2):87-93.

15. Seidl EMF, Melchíades A, Farias V, Brito A. Pessoas vivendo com HIV/aids: variáveis associadas à adesão ao tratamento antirretroviral. Cad Saúde Pública 2007;23(10): 2305-16.

16. Ministério da Saúde. Programa Nacional de DST e Aids. Manual de Adesão ao Tratamento: para pessoas vivendo com HIV e Aids. Brasília, DF: Ministério da Saúde; 2008.

17. Torres TL, Camargo BV. Representações sociais da Aids e da Terapia Antirretroviral para pessoas vivendo com HIV. Psicol Teor Prática 2008;10(1):64-78.

18. Feitosa AC, Lima HJA, Caetano JA, Andrade LM, Beserra EP. Terapia anti-retroviral: fatores que interferem na Adesão de crianças com HIV/AIDS. Esc Anna Nery Rev Enferm 2008;12(3):515-21.

19. Schilkowsky LB, Portela MC, Sa MC.Fatores associados ao abandono de acompanhamento ambulatorial em um serviço de assistência especializada em HIV/aids na cidade do Rio de Janeiro, RJ. Rev Bras Epidemiol 2011;14(2):187-197

20. Paula CC, Cabral IE, Souza IEO. O cotidiano do ser-adolescendo que tem aids: momento ou movimento existencial? Esc Anna Nery Rev Enferm 2009;13(3):632-639.

21. Martins SS, Martins TSS. Adesão ao tratamento antirretroviral: vivências de escolares. Texto \& Contexto Enferm 2011;20(1):111-8.

22. Marques HHS, Silva NG, Gutierrez PL, Lacerda R, Ayres JRCM, Dellanegra $M$, et al. A revelação do diagnóstico na perspectiva dos adolescentes vivendo com HIV/aids e seus pais e cuidadores. Cad Saúde Pública 2006;22(3):619-29.

23. Lazzarotto AR, Reichert MT, Venker CA, Kramer AS, Sprinz E. HIV/aids e meia idade: avaliação do conhecimento de indivíduos da região do Vale do Sinos (RS), Brasil. Ciênc Saúde Coletiva 2010;15(Suppl.1):1185-1190.

24. Cardoso AL, Marcon SS, Waidmani MAP. O impacto da descoberta da sorologia positiva do portador de HIV/AIDS e sua família. Rev Enferm UERJ 2008;16(3):326-332. 